\title{
Nanoindentation, EPMA and Atom Probe Tomography Characterisation of Oxygen-rich Layer formed on a Titanium Jet Engine Component
}

\author{
HM Gardner $^{1 *}$, A Radecka ${ }^{2}$, D Rugg ${ }^{2}$, DEJ Armstrong ${ }^{1}$, MP Moody ${ }^{1}$ and PAJ Bagot ${ }^{1}$ \\ 1. Department of Materials, University of Oxford, Oxford, United Kingdom. \\ 2. Rolls-Royce plc, Derby, United Kingdom. \\ * Corresponding author: hazel.gardner@materials.ox.ac.uk
}

Titanium alloys are widely used in the aerospace industry owing to their high specific strength to weight ratio. Within an aeroengine, titanium is predominantly used at the cooler front end in the form of fan blades and compressor discs. The use of titanium in an aeroengine is maximised as far as possible to minimise total engine weight. However, oxygen degrades the high temperature performance of titanium. Temperatures above $480^{\circ} \mathrm{C}$ enable large amounts of oxygen to diffuse into the surface of titanium components, forming a brittle, oxygen-rich surface layer known as 'alpha case', which is detrimental to mechanical properties [1]. Thus critical rotating parts beyond the compressor are made from materials with superior high temperature performance, such as nickel superalloys, despite nickel being heavier than titanium. Alpha case formation has potential to occur both during alloy processing and in service, limiting the maximum operating temperature of titanium alloys. Better understanding of the alpha case layer is needed so that the use of titanium alloys at higher temperatures can be safely explored, with the aim of reducing total engine weight.

Recent work [2] has shown that nanoscale features exist within the alpha case layer, highlighting the need for characterisation techniques with high spatial resolution. In this study, the alpha case layer formed on a section of IMI 834 compressor disc as a result of environmental exposure was characterised across a range of length scales through complementary use of nanoindentation, electron probe microanalysis (EPMA) and atom probe tomography (APT).

A section of compressor disc, provided by Rolls-Royce plc, was mounted in conductive resin and prepared using conventional metallographic polishing methods to allow nanoindentation and EPMA depth profiles to be performed on a cross section through the alpha case layer (figure 1). APT samples were also prepared from this cross section at increasing depths into the alpha case layer, using the standard focussed ion beam lift out method.

Nanohardness profiles taken across the alpha case region are compared the shape of the semi-quantitative EPMA oxygen profile through the alpha case. The effect of crystallography and microstructure on nanohardness has also been explored. Extent of oxygen diffusion from the surface into the bulk is commonly inferred using hardness profiles and semi-quantitative EPMA oxygen profiles [3]. However, in this study the oxygen content as a function of depth has been directly quantified using APT, and compared to the shape of the EPMA oxygen profile. APT has also revealed nanoscale microstructural features within the alpha case (figure 2).

In conclusion, we have presented the first steps in the process of full characterisation of the alpha case layer formed on an IMI 834 compressor disc. We have quantified oxygen content as a function of depth into the alpha case and, when combined with complementary nanohardness and EPMA oxygen profiles, this information can be used to report an alpha case depth, as defined by depth of oxygen diffusion. 
References:

[1] R Gaddam et al., IOP Conf. Ser. Mater. Sci. Eng. 48 (2013), p. 012002.

[2] PAJ Bagot et al., Scr. Mater.148 (2018).

[3] R Gaddam et al., Mater. Charact. 99 (2015), p. 166.

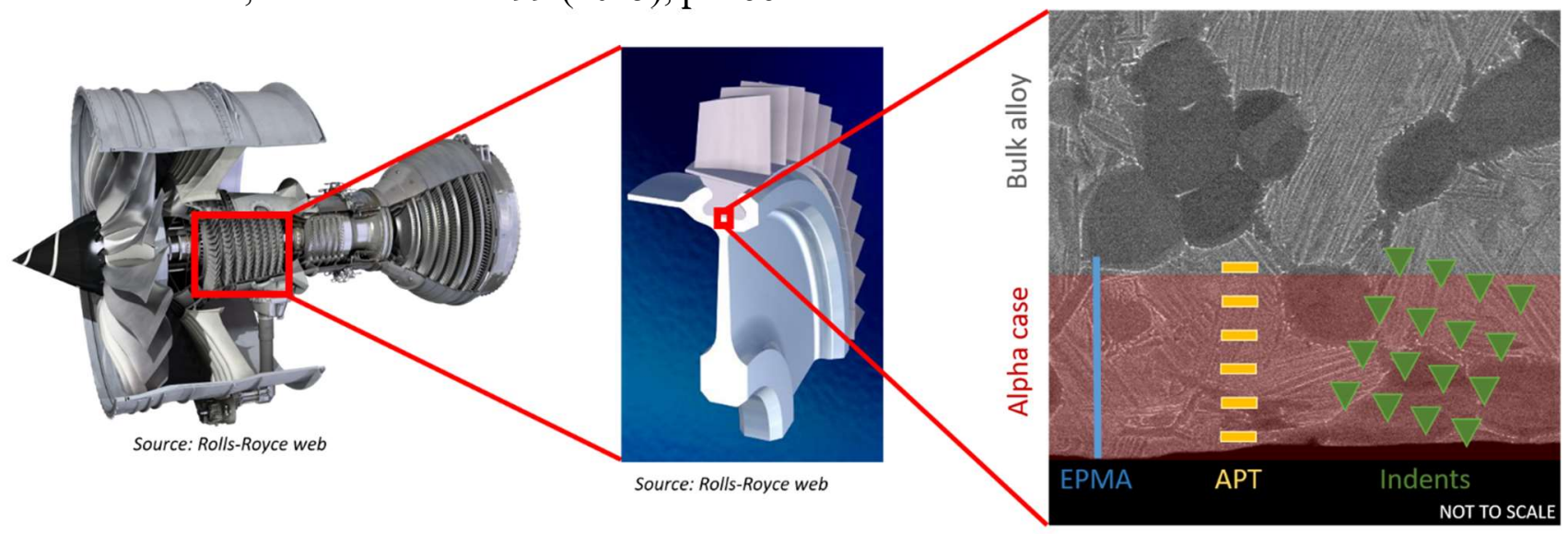

Jet engine

Compressor disc

Schematic of main analysis performed

Figure 1. Illustration showing the origin of material in this study, and a schematic of the main analysis performed.

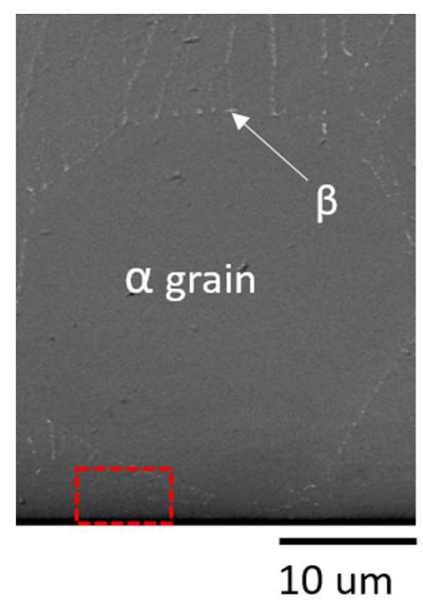

(a)

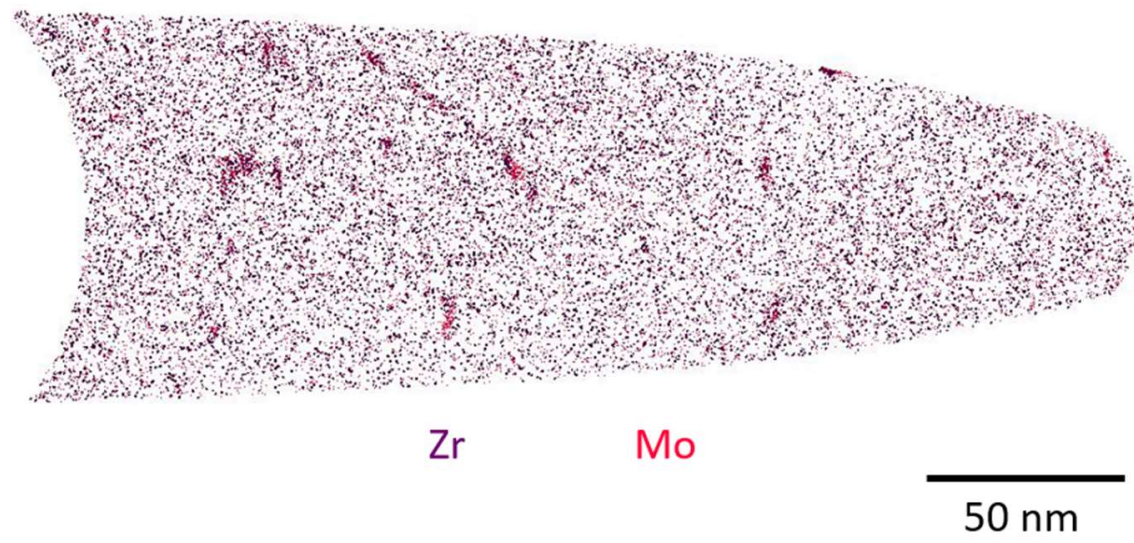

(b)

Figure 2. (a) SEM image of part of alpha case region formed on IMI 834 compressor disc. Surface of compressor disc is at bottom of image. Dotted line indicates area from which the APT sample shown in (b) was taken. Atom map in (b) shows nanoscale segregation of $\mathrm{Zr}$ and Mo. 